\title{
Investigation of the Effects of Heat Treatment on Varnished Wood Material
}

\author{
Beytullah Kazan 1(D), Murat Özalp ${ }^{\text {**iD }}$
}

\begin{abstract}
In this study, the effects of the heat treatment applied varnished wood materials on hardness, brightness and varnishes' surface sticking resistance were investigated. For this purpose, firstly Pinus sylvestris L., Fagus orientalis L. and Castanea sativa M. wooden samples were varnished by the water based varnish and after that they were kept in temperatures of $100{ }^{\circ} \mathrm{C}, 125^{\circ} \mathrm{C}$ and $150{ }^{\circ} \mathrm{C}$ for times of 2,4 and 6 hours. According to test results it was determined that while the hardness and varnishes' surface sticking resistance were improved for all samples which were processed 2 hours in temperatures of $100{ }^{\circ} \mathrm{C}$, the brightness, surface sticking resistance and brightness of the samples which were processed 4 and 6 hours in $100{ }^{\circ} \mathrm{C}$ and 2,4 and 6 hours in $125^{\circ} \mathrm{C}$ and $150{ }^{\circ} \mathrm{C}$ were deteriorated. Furthermore, it was observed that the hardness resistance of all samples which processed for 2,4 and 6 hours in $100{ }^{\circ} \mathrm{C}, 125^{\circ} \mathrm{C}$ and $150{ }^{\circ} \mathrm{C}$ were high. In all varnished wood species, the dual-component varnish gave better results than the single-component varnish.
\end{abstract}

Keywords: Heat treatment, hardness, brightness, resistance of sticking, water-based varnish.

1Address: Kutahya Dumlupinar University, Faculty of Simav Technology, Department of Wood Products Industrial Engineering, 43500 Simav-Kütahya/Turkey

*Corresponding author: murat.ozalp@dpu.edu.tr

Citation: Kazan, B., Özalp, M. (2021). Investigation of the Effects of Heat Treatment on Varnished Wood Material. Bilge International Journal of Science and Technology Research, 5 (1): 22-33.

\section{INTRODUCTION}

Top surface processes have been applied since time immemorial in order to provide protecting against external effects, to increase esthetical value, and to eliminate negative properties of wood material (San1var,2001). A range of chemical changes and reactions resulting in degradation of components such as wood hemicellulose, lignin etc. by the effect of high temperature during heat treatments occur, and changes in wood structure are formed. It is applied temperatures over $150{ }^{\circ} \mathrm{C}$ for places of use desired high durability, and under $150{ }^{\circ} \mathrm{C}$ for indoor usages generally (Kantay and Kartal,2007). Water vapor and high temperatures in heat treatment processes are used in principle. Therefore, process conditions have corrosive properties and water and various components are dissociate from wood and are evaporated (Thermowood,2003). Budakci, investigated the effect of layer thickness on hardness, brightness, and surface sticking resistance in wood varnishes. He determined that third layer varnish applications don't have any effect on hardness and increasing layer thickness increases surface sticking resistance in polymeric-based varnishes (Budakc1,1997).
Highley and Kicle, reported that coating with different varnish layer on the purpose of protecting surface of wood materials against external effects is a method used as most commonly (Highley and Kicle, 1990). Atar, performed bleaching with 6 group solutions in impregnated and natural scotch pine, oriental beech, chestnut and sessile oak woods. It was applied water-based and synthetic varnish to their surfaces, and it was specified that bleaching tools decrease surface sticking resistance of varnishes by $3-5 \%$ averagely, and the best results were obtained with waterbased varnish (Atar,1999). Sogutlu, determined that wood material high density is also have a high surface sticking resistance, as a result of regression analysis performed to specify relation between density and adhesive resistance (Sogutlu,2004). The pentahydrate added into the varnish increased toughness and sticking resistance; however, it decreased the brightness rate of varnish on the other side (Ozalp, 2008). It is observed that as the rate of the pentahydrate added into the water-based varnish increased from $0 \%$ to $30 \%$, the varnishes' sticking rate increased $50.46 \%$ in Chestnut wood and $37.04 \%$ in Scotch pine wood. Toughness rate increased $55.79 \%$ in Chestnut wood and $41.98 \%$ in Scotch pine, Brightness rate decreased 
$68.52 \%$ in Chestnut wood and $58.88 \%$ in Scotch pine (Ozalp and Korkut, 2001).

The purpose of the study is to determine effects of heat treatment applied to varnished wood material on properties of varnish.

\section{MATERIAL VE METHOD}

\subsection{Wood Material}

First-class and flawless wood samples obtained from scotch pine (Pinus silvestris L.), beech wood (Fagus orientalis L.), chestnut (Castanea sativa M.) woods were used in all experiments. Experiment samples were prepared according to ASTM-D 358 and TS 801 principals (ASTM-D $358,1998)$. After samples in air-dry moisture were brought to $100 \times 100 \times 8$ dimension, they were sanded with 100 numbered sandpaper first,and 120 numbered sandpaper after. 300 samples were prepared as 5 experiments for every tree species, varnish species, and heat treatment method in the study.

\subsection{Varnish}

In varnishing of experiment samples, (A) single component belong to Trimetal company and (B) dual component belong to Hemel company (Sayerlack VS 5341+ AH 1547 hardener) water-based varnishes were used. It was corresponded to ASTM D-3023 principals in varnishing of samples. Properties of varnishes used in experiments were given in Table1.

Table 1. Technical specifications of varnishes

\begin{tabular}{|l|l|l|}
\hline \multirow{2}{*}{ Specifications } & \multicolumn{2}{|l|}{ Varnish type } \\
\cline { 2 - 3 } & $\begin{array}{l}\text { Single } \\
\text { components }\end{array}$ & $\begin{array}{l}\text { Dual } \\
\text { components }\end{array}$ \\
\hline Amounts of solids $(\%)$ & $30 \pm 2$ & $36 \pm 1$ \\
\hline $\mathrm{P}^{\mathrm{H}}\left(25^{\circ} \mathrm{C}\right)$ & 8,8 & 9,2 \\
\hline Layer thickness $(\mu \mathrm{m})$ & 85 & 130 \\
\hline
\end{tabular}

\subsection{Heat Treatment Application}

Heat treatment was applied to varnished materials and in air-dry conditions. Experiments were subjected to heat treatment inside oven in $100{ }^{\circ} \mathrm{C}, 125{ }^{\circ} \mathrm{C}$ and $150{ }^{\circ} \mathrm{C}$ for times of 2, 4, 6 hours in conformity with the purpose of experiment.

\subsection{Adhesive}

In experiments, 404 Steel Adhesive, which doesn't have solver effect on two-component epoxy-resinous varnish layers according to ASTM D-4541 and TS EN-24624 and have high adhesive force was used ASTM D-4541,1995; TS EN-24624, 1996)].

\subsection{Surface Sticking Test}

Surface sticking resistance of varnish layers was determined in adhesion test device working with air system by adapting to ASTM D-4541 and TS EN 24624 principals in the research ((ASTM D-4541,1995; TS EN-24624, 1996). The equation given below was used while calculating the resistance of sticking.

$X=4 \mathrm{~F} / \pi \cdot \mathrm{d}^{2}$

Where;

F: Splitting force (Newton), d: Sample cylinder's radius $(\mathrm{mm}), \mathrm{X}$ : Sticking resistance (MPa).

\subsection{Pendulum Hardness Measurement}

Hardness values of varnish layer specifying durability to external factors were determined with pendulum hardness measurement device according to könig method by adapting to principals specified in ANS/ISO 1522 (ANS/ISO 1522,1998).

\subsection{Surface Brightness Measurement}

Measurements were performed with brightness measurement device (Glossmeter) by taking advantage of shining capability of varnish surfaces within the frame of principals specified in TS 4318 EN ISO 2813 (TS 4318 EN ISO 2813,2002).

\section{RESULTS}

\subsection{Hardness Resistance Measurements}

Obtained hardness resistance values belong to scotch pine wood were given in Table 2. 
Table 2. Hardness resistance values belong to scotch pine wood

\begin{tabular}{|c|c|c|c|c|c|c|c|c|c|c|}
\hline \multicolumn{11}{|c|}{ Hardness measurement values (Pendulum) } \\
\hline $\begin{array}{l}\text { Wood } \\
\text { type }\end{array}$ & Temperature & Time & Varnish & \multicolumn{5}{|c|}{ Values } & $\mathrm{n}$ & Average \\
\hline \multirow{20}{*}{ 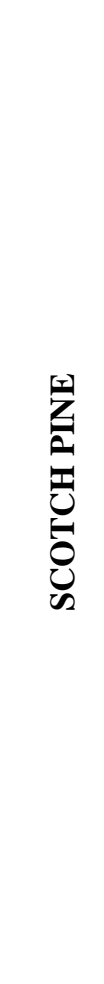 } & \multirow{2}{*}{ Non-treatment } & \multirow{2}{*}{ - } & $\mathrm{A}$ & 33 & 35 & 35 & 31 & 34 & 5 & 33,6 \\
\hline & & & $\mathrm{B}$ & 48 & 37 & 44 & 44 & 39 & 5 & 42,3 \\
\hline & \multirow{6}{*}{ g } & \multirow[b]{2}{*}{2} & A & 30 & 36 & 36 & 34 & 37 & 5 & 34,6 \\
\hline & & & $\mathrm{B}$ & 48 & 42 & 47 & 43 & 41 & 5 & 44,2 \\
\hline & & \multirow[b]{2}{*}{4} & A & 31 & 35 & 34 & 36 & 38 & 5 & 34,8 \\
\hline & & & B & 47 & 45 & 44 & 45 & 46 & 5 & 45,4 \\
\hline & & \multirow[b]{2}{*}{6} & A & 38 & 36 & 39 & 36 & 35 & 5 & 36,8 \\
\hline & & & B & 48 & 48 & 47 & 46 & 46 & 5 & 47 \\
\hline & \multirow{6}{*}{$\stackrel{1}{-1}$} & \multirow[b]{2}{*}{2} & A & 34 & 36 & 35 & 35 & 38 & 5 & 35,6 \\
\hline & & & B & 46 & 48 & 47 & 47 & 49 & 5 & 47,4 \\
\hline & & \multirow[b]{2}{*}{4} & A & 35 & 35 & 36 & 38 & 39 & 5 & 36,6 \\
\hline & & & B & 48 & 48 & 50 & 51 & 49 & 5 & 49,2 \\
\hline & & \multirow[b]{2}{*}{6} & A & 36 & 38 & 36 & 37 & 38 & 5 & 37 \\
\hline & & & B & 50 & 51 & 48 & 49 & 52 & 5 & 50 \\
\hline & \multirow{6}{*}{ 복 } & \multirow[b]{2}{*}{2} & A & 36 & 38 & 36 & 34 & 38 & 5 & 36,4 \\
\hline & & & $\mathrm{B}$ & 50 & 51 & 49 & 49 & 52 & 5 & 50,2 \\
\hline & & \multirow[b]{2}{*}{4} & A & 38 & 38 & 40 & 36 & 38 & 5 & 38 \\
\hline & & & B & 48 & 51 & 52 & 52 & 54 & 5 & 51,4 \\
\hline & & \multirow[b]{2}{*}{6} & A & 39 & 38 & 36 & 39 & 40 & 5 & 38,4 \\
\hline & & & B & 54 & 52 & 50 & 54 & 51 & 5 & 52,2 \\
\hline
\end{tabular}

After single and dual component varnish applications of scotch pine tree; lowest hardness values were seen in specimens subjected to non-heat-treated single component varnish application, while highest hardness values were seen in specimens subjected to dual component varnish application and waited for 6 hours in $150{ }^{\circ} \mathrm{C}$ as a result of comparing hardness values of non-heat-treated specimens and heat-treated specimens according to heat treatment temperature and time. As a result of comparing hardness values of non-heat-treated specimens subjected to single component varnish application and heat-treated specimens, it was seen that heat-treated specimens have higher hardness values compared with specimens varnished without heat-treatment. As a result of comparing dual component varnished non-heat-treated specimens and heattreated specimens, it was observed that hardness values of heat-treated specimens have higher than specimens varnished without heat-treatment.

Obtained hardness resistance values belong to beech wood were given in Table 3 . 
Table 3. Hardness resistance values belong to beech wood

\begin{tabular}{|c|c|c|c|c|c|c|c|c|c|c|}
\hline \multicolumn{11}{|c|}{ Hardness measurement values (Pendulum) } \\
\hline $\begin{array}{l}\text { Wood } \\
\text { type }\end{array}$ & Temperature & $\begin{array}{l}\text { Time } \\
\text { (hour) }\end{array}$ & Varnish & \multicolumn{5}{|c|}{ Values } & $\mathrm{n}$ & Average \\
\hline \multirow{20}{*}{ 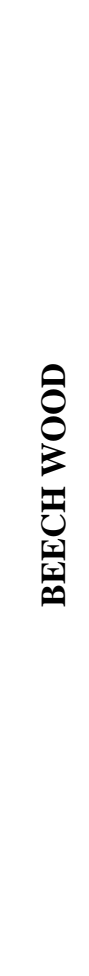 } & \multirow{2}{*}{ Non-treatment } & \multirow{2}{*}{ - } & A & 49 & 53 & 60 & 60 & 54 & 5 & 55,2 \\
\hline & & & B & 61 & 58 & 66 & 61 & 63 & 5 & 61,8 \\
\hline & \multirow{6}{*}{$\Xi$} & \multirow{2}{*}{2} & A & 54 & 58 & 53 & 58 & 57 & 5 & 56 \\
\hline & & & B & 68 & 66 & 65 & 60 & 59 & 5 & 63,6 \\
\hline & & \multirow{2}{*}{4} & A & 56 & 58 & 59 & 57 & 58 & 5 & 57,6 \\
\hline & & & B & 65 & 66 & 65 & 63 & 63 & 5 & 64,4 \\
\hline & & \multirow{2}{*}{6} & A & 56 & 59 & 60 & 58 & 57 & 5 & 58 \\
\hline & & & B & 65 & 63 & 68 & 64 & 67 & 5 & 65,4 \\
\hline & \multirow{6}{*}{$\stackrel{2}{3}$} & \multirow{2}{*}{2} & A & 56 & 59 & 60 & 59 & 59 & 5 & 58,6 \\
\hline & & & B & 65 & 64 & 65 & 67 & 68 & 5 & 65,8 \\
\hline & & \multirow{2}{*}{4} & A & 58 & 60 & 61 & 59 & 58 & 5 & 59,2 \\
\hline & & & B & 68 & 64 & 69 & 67 & 68 & 5 & 67,2 \\
\hline & & \multirow{2}{*}{6} & A & 60 & 61 & 59 & 61 & 58 & 5 & 59,8 \\
\hline & & & B & 68 & 65 & 70 & 69 & 68 & 5 & 68 \\
\hline & \multirow{6}{*}{ ำ } & \multirow{2}{*}{2} & A & 58 & 61 & 60 & 58 & 58 & 5 & 59 \\
\hline & & & B & 69 & 69 & 65 & 68 & 68 & 5 & 67,8 \\
\hline & & \multirow{2}{*}{4} & A & 58 & 61 & 62 & 58 & 60 & 5 & 59,8 \\
\hline & & & B & 68 & 69 & 71 & 68 & 68 & 5 & 68,8 \\
\hline & & \multirow{2}{*}{6} & A & 59 & 60 & 62 & 62 & 63 & 5 & 61,2 \\
\hline & & & B & 68 & 70 & 70 & 71 & 71 & 5 & 70 \\
\hline
\end{tabular}

After single and dual component varnish applications of beech wood; lowest hardness values were seen in non-heattreated specimens, while highest hardness values were seen in dual component varnished specimens waited for 6 hours in $150{ }^{\circ} \mathrm{C}$ according to heat treatment temperature and time conditions. As a result of comparing hardness values of non-heat-treated specimens subjected to single component varnish application and heat-treated specimens, it was seen that heat-treated specimens have higher hardness values compared with specimens varnished as non-heat-treated. As a result of comparing dual component varnished non-heattreated specimens and heat-treated specimens, it was observed that hardness values of heat-treated specimens have higher than specimens varnished without heattreatment.

Obtained hardness measurement values belong to chestnut wood were given in Table 4. 
Table 1. Hardness measurement values belong to chestnut wood

\begin{tabular}{|c|c|c|c|c|c|c|c|c|c|c|}
\hline \multicolumn{11}{|c|}{ Hardness measurement values (Pendulum) } \\
\hline Wood & Temperature & Time & Varnish & \multicolumn{5}{|c|}{ Values } & $\mathrm{n}$ & Average \\
\hline \multirow{20}{*}{ 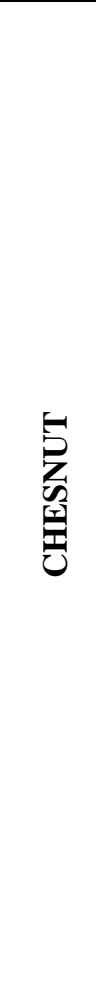 } & \multirow{2}{*}{ Non-treatment } & \multirow{2}{*}{ - } & A & 43 & 39 & 51 & 38 & 42 & 5 & 42,6 \\
\hline & & & $\mathrm{B}$ & 59 & 63 & 52 & 49 & 53 & 5 & 55,2 \\
\hline & \multirow{6}{*}{$\stackrel{8}{\varrho}$} & \multirow{2}{*}{2} & A & 49 & 45 & 40 & 40 & 44 & 5 & 43,6 \\
\hline & & & $\mathrm{B}$ & 60 & 58 & 58 & 56 & 59 & 5 & 58,2 \\
\hline & & \multirow{2}{*}{4} & A & 45 & 47 & 48 & 46 & 41 & 5 & 45,4 \\
\hline & & & B & 59 & 64 & 60 & 60 & 57 & 5 & 60 \\
\hline & & \multirow{2}{*}{6} & A & 46 & 48 & 48 & 47 & 46 & 5 & 47 \\
\hline & & & B & 61 & 62 & 59 & 63 & 61 & 5 & 61,2 \\
\hline & \multirow{6}{*}{ 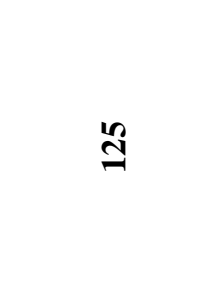 } & \multirow{2}{*}{2} & A & 48 & 44 & 45 & 47 & 48 & 5 & 46,4 \\
\hline & & & B & 63 & 61 & 59 & 62 & 62 & 5 & 61,4 \\
\hline & & \multirow{2}{*}{4} & A & 46 & 48 & 47 & 48 & 46 & 5 & 47 \\
\hline & & & B & 62 & 64 & 62 & 62 & 63 & 5 & 62,6 \\
\hline & & \multirow{2}{*}{6} & A & 48 & 49 & 48 & 50 & 47 & 5 & 48,4 \\
\hline & & & B & 65 & 63 & 63 & 62 & 65 & 5 & 63,6 \\
\hline & \multirow{6}{*}{ 복 } & \multirow{2}{*}{2} & A & 45 & 48 & 47 & 50 & 51 & 5 & 48,2 \\
\hline & & & B & 65 & 62 & 63 & 66 & 64 & 5 & 64 \\
\hline & & \multirow{2}{*}{4} & A & 50 & 48 & 49 & 51 & 47 & 5 & 49 \\
\hline & & & B & 65 & 62 & 67 & 67 & 64 & 5 & 65 \\
\hline & & \multirow{2}{*}{6} & A & 51 & 49 & 50 & 50 & 50 & 5 & 50 \\
\hline & & & B & 68 & 69 & 63 & 65 & 67 & 5 & 66,4 \\
\hline
\end{tabular}

After single and dual component varnish applications of chestnut wood; lowest hardness values were seen in nonheat-treated single component specimens, while highest hardness values were seen in dual component varnished specimens waited for 6 hours in $150{ }^{\circ} \mathrm{C}$ according to heat treatment temperature and time conditions. As a result of comparing hardness values of non-heat-treated specimens subjected to single component varnish application and heattreated specimens, it was observed that heat-treated specimens have higher hardness values compared with specimens varnished as non-heat-treated. As a result of comparing dual component varnished non-heat-treated specimens and heat-treated specimens, it was seen that hardness values of heat-treated specimens have higher than specimens varnished without heat-treatment.

\subsection{The results of varnishes' surface sticking resistance}

Obtained varnishes' surface sticking resistance values belong to scotch pine wood were given in Table 5 . 
Table 5. Varnishes' surface sticking resistance values belong to scotch pine wood

\begin{tabular}{|c|c|c|c|c|c|c|c|c|c|c|}
\hline \multicolumn{11}{|c|}{ Surface sticking resistance (MPa) } \\
\hline $\begin{array}{l}\text { Wood } \\
\text { type }\end{array}$ & $\begin{array}{c}\text { Temperature } \\
\left({ }^{\circ} \mathrm{C}\right)\end{array}$ & $\begin{array}{l}\text { Time } \\
\text { (hour) }\end{array}$ & $\begin{array}{c}\text { Varnish } \\
\text { type }\end{array}$ & \multicolumn{5}{|c|}{ Values } & $\mathrm{n}$ & Average \\
\hline \multirow{20}{*}{ 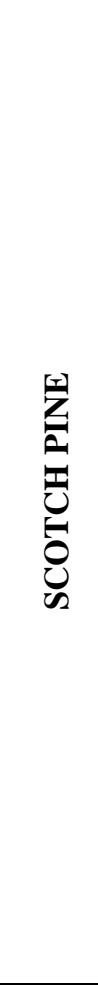 } & \multirow{2}{*}{ Non-treatment } & \multirow[b]{2}{*}{-} & A & 3,82 & 3,56 & 3,63 & 3,37 & 3,43 & 5 & 3,56 \\
\hline & & & $\mathrm{B}$ & 4,14 & 3,94 & 4,14 & 3,75 & 3,82 & 5 & 3,95 \\
\hline & \multirow{6}{*}{$\stackrel{乛}{\varrho}$} & \multirow{2}{*}{2} & A & 3,82 & 3,69 & 3,56 & 3,63 & 3,75 & 5 & 3,69 \\
\hline & & & $\mathrm{B}$ & 4,33 & 4,33 & 4,39 & 4,14 & 4,20 & 5 & 4,27 \\
\hline & & \multirow{2}{*}{4} & A & 3,63 & 3,63 & 3,56 & 3,69 & 3,69 & 5 & 3,64 \\
\hline & & & $\mathrm{B}$ & 4,33 & 4,26 & 4,20 & 4,07 & 4,14 & 5 & 4,20 \\
\hline & & \multirow{2}{*}{6} & A & 3,63 & 3,56 & 3,56 & 3,69 & 3,43 & 5 & 3,57 \\
\hline & & & B & 4,07 & 4,07 & 4,33 & 4,14 & 4,01 & 5 & 4,12 \\
\hline & \multirow{6}{*}{$\stackrel{1}{\stackrel{1}{J}}$} & \multirow{2}{*}{2} & A & 3,43 & 3,37 & 3,56 & 3,63 & 3,56 & 5 & 3,51 \\
\hline & & & $\mathrm{B}$ & 4,20 & 4,14 & 4,01 & 3,88 & 4,01 & 5 & 4,04 \\
\hline & & \multirow{2}{*}{4} & A & 3,37 & 3,43 & 3,56 & 3,43 & 3,37 & 5 & 3,43 \\
\hline & & & $\mathrm{B}$ & 3,94 & 4,07 & 3,82 & 3,94 & 4,01 & 5 & 3,95 \\
\hline & & \multirow{2}{*}{6} & A & 3,50 & 3,31 & 3,50 & 3,37 & 3,31 & 5 & 3,39 \\
\hline & & & B & 3,75 & 4,01 & 3,82 & 3,88 & 4,01 & 5 & 3,89 \\
\hline & \multirow{6}{*}{$\stackrel{n}{n}$} & \multirow{2}{*}{2} & $\mathrm{~A}$ & 3,56 & 3,37 & 3,43 & 3,31 & 3,31 & 5 & 3,39 \\
\hline & & & $\mathrm{B}$ & 3,94 & 3,82 & 3,75 & 4,01 & 3,94 & 5 & 3,89 \\
\hline & & \multirow{2}{*}{4} & A & 3,43 & 3,31 & 3,24 & 3,18 & 3,43 & 5 & 3,31 \\
\hline & & & B & 3,69 & 3,82 & 3,88 & 3,75 & 3,94 & 5 & 3,81 \\
\hline & & \multirow{2}{*}{6} & A & 3,05 & 3,18 & 3,31 & 3,24 & 3,12 & 5 & 3,18 \\
\hline & & & B & 3,82 & 3,69 & 3,75 & 3,63 & 3,88 & 5 & 3,75 \\
\hline
\end{tabular}

After single and dual component varnish applications of scotch pine wood; lowest adhesive resistance was seen in specimens performed single component varnish application and waited for 6 hours in $150{ }^{\circ} \mathrm{C}$, while highest adhesive resistance was seen in specimens performed dual component varnish application and waited for 2 hours in $100{ }^{\circ} \mathrm{C}$ according to heat treatment temperature and time conditions. As a result of comparing adhesive resistance values of non-heat-treated specimens subjected to single component varnish application and heat-treated specimens, specimens waited for $2,4,6$ hours in $100{ }^{\circ} \mathrm{C}$ have higher adhesive resistance than specimens varnished as without heat treatment, and it was seen that adhesive resistance values decreased in other heat treatment temperatures and times. As a result of comparing dual component varnished non-heat-treated specimens and heat-treated specimens, specimens waited for $2,4,6$ hours in $100{ }^{\circ} \mathrm{C}$ and waited for 2 hours in $125{ }^{\circ} \mathrm{C}$ have higher adhesive resistance, and it was observed that adhesive resistance values decreased in other heat treatment temperatures and times.

Obtained varnishes' surface sticking resistance values belong to beech wood were given in Table 6 . 
Table 6. Varnishes' surface sticking resistance values belong to beech wood

\begin{tabular}{|c|c|c|c|c|c|c|c|c|c|c|}
\hline \multicolumn{11}{|c|}{ Surface sticking resistance (MPa) } \\
\hline $\begin{array}{l}\text { Wood } \\
\text { type }\end{array}$ & $\begin{array}{l}\text { Temperature } \\
\left({ }^{\circ} \mathrm{C}\right)\end{array}$ & $\begin{array}{l}\text { Time } \\
\text { (hour) }\end{array}$ & $\begin{array}{l}\text { Varnish } \\
\text { type }\end{array}$ & \multicolumn{5}{|c|}{ Values } & $\mathrm{n}$ & Average \\
\hline \multirow{20}{*}{$\begin{array}{l}0 \\
0 \\
0 \\
0 \\
0 \\
0 \\
0 \\
0 \\
0 \\
0\end{array}$} & \multirow{2}{*}{ Non-treatment } & \multirow[b]{2}{*}{-} & A & 5,28 & 4,96 & 5,35 & 5,09 & 5,09 & 5 & 5,14 \\
\hline & & & $\mathrm{B}$ & 5,73 & 5,54 & 5,60 & 5,79 & 5,54 & 5 & 5,64 \\
\hline & \multirow{6}{*}{$\stackrel{8}{\varrho}$} & \multirow{2}{*}{2} & $\mathrm{~A}$ & 5,47 & 5,41 & 5,28 & 5,41 & 5,54 & 5 & 5,42 \\
\hline & & & $\mathrm{B}$ & 5,86 & 5,73 & 5,47 & 5,60 & 5,47 & 5 & 5,62 \\
\hline & & \multirow{2}{*}{4} & A & 5,47 & 5,41 & 5,35 & 5,35 & 5,28 & 5 & 5,37 \\
\hline & & & $\mathrm{B}$ & 5,60 & 5,41 & 5,66 & 5,54 & 5,41 & 5 & 5,52 \\
\hline & & \multirow{2}{*}{6} & $\mathrm{~A}$ & 5,35 & 5,28 & 5,35 & 5,28 & 5,15 & 5 & 5,28 \\
\hline & & & $\mathrm{B}$ & 5,35 & 5,47 & 5,54 & 5,41 & 5,35 & 5 & 5,42 \\
\hline & \multirow{6}{*}{$\stackrel{1}{\stackrel{1}{7}}$} & \multirow{2}{*}{2} & A & 5,15 & 5,09 & 5,28 & 5,28 & 5,03 & 5 & 5,16 \\
\hline & & & B & 5,35 & 5,41 & 5,35 & 5,54 & 5,47 & 5 & 5,42 \\
\hline & & \multirow{2}{*}{4} & A & 5,22 & 5,03 & 5,03 & 4,96 & 5,28 & 5 & 5,10 \\
\hline & & & $\mathrm{B}$ & 5,35 & 5,28 & 5,47 & 5,15 & 5,22 & 5 & 5,29 \\
\hline & & \multirow{2}{*}{6} & A & 4,96 & 4,96 & 4,84 & 5,09 & 5,03 & 5 & 4,97 \\
\hline & & & $\mathrm{B}$ & 5,28 & 5,15 & 5,28 & 5,09 & 5,28 & 5 & 5,21 \\
\hline & \multirow{6}{*}{ 옥 } & \multirow{2}{*}{2} & A & 4,90 & 4,96 & 5,09 & 4,96 & 5,03 & 5 & 4,98 \\
\hline & & & $\mathrm{B}$ & 5,09 & 5,28 & 5,15 & 5,35 & 5,28 & 5 & 5,23 \\
\hline & & \multirow{2}{*}{4} & A & 4,84 & 4,77 & 4,96 & 4,84 & 4,96 & 5 & 4,87 \\
\hline & & & $\mathrm{B}$ & 5,22 & 5,15 & 5,03 & 5,09 & 4,96 & 5 & 5,09 \\
\hline & & \multirow{2}{*}{6} & A & 4,77 & 4,96 & 4,84 & 4,84 & 4,71 & 5 & 4,82 \\
\hline & & & B & 5,03 & 4,96 & 5,09 & 5,03 & 4,96 & 5 & 5,01 \\
\hline
\end{tabular}

After single and dual component varnish applications of beech wood; lowest adhesive resistance was seen in specimens performed single component varnish application and waited for 6 hours in $150{ }^{\circ} \mathrm{C}$, while highest adhesive resistance was seen in specimens performed dual component varnish application and waited for 2 hours in $100{ }^{\circ} \mathrm{C}$ according to heat treatment temperature and time conditions. As a result of comparing adhesive resistance values of non-heat-treated specimens subjected to single component varnish application and heat-treated specimens, specimens waited for $2,4,6$ hours in $100{ }^{\circ} \mathrm{C}$ and waited for 2 hours in $125^{\circ} \mathrm{C}$ have higher adhesive resistance compared to specimens varnished as without heat treatment. As a result of comparing dual component varnished non-heattreated specimens and heat-treated specimens, specimens waited for 2, 4, 6 hours in $100{ }^{\circ} \mathrm{C}$ and waited for 2 hours in $125{ }^{\circ} \mathrm{C}$ have higher adhesive resistance, and it was observed that adhesive resistance values decreased in other heat treatment temperatures and times.

Obtained varnishes' surface sticking resistance values belong to chestnut wood were given in Table 7 . 
Table 7. Varnishes' surface sticking resistance values belong to chestnut wood

\begin{tabular}{|c|c|c|c|c|c|c|c|c|c|c|}
\hline \multicolumn{11}{|c|}{ Surface sticking resistance $(\mathrm{MPa})$} \\
\hline $\begin{array}{l}\text { Wood } \\
\text { type }\end{array}$ & \multirow{3}{*}{$\begin{array}{l}\begin{array}{l}\text { Temperature } \\
\left({ }^{\circ} \mathrm{C}\right)\end{array} \\
\text { Non-treatment }\end{array}$} & \multirow{3}{*}{$\begin{array}{l}\text { Time } \\
\text { (hour) } \\
-\end{array}$} & \multirow{2}{*}{$\begin{array}{l}\text { Varnish } \\
\text { type } \\
\text { A }\end{array}$} & \multicolumn{5}{|c|}{ Values } & \multirow{2}{*}{\begin{tabular}{|l}
$\mathrm{n}$ \\
5
\end{tabular}} & \multirow{2}{*}{$\begin{array}{l}\text { Average } \\
3,84\end{array}$} \\
\hline \multirow{20}{*}{ 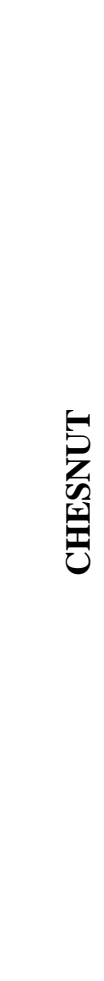 } & & & & 3,88 & 3,69 & 3,82 & 3,88 & 3,94 & & \\
\hline & & & $\mathrm{B}$ & 4,14 & 4,20 & 4,45 & 4,33 & 4,39 & 5 & 4,30 \\
\hline & \multirow{6}{*}{ छ } & \multirow{2}{*}{2} & $\mathrm{~A}$ & 4,07 & 4,14 & 4,07 & 4,01 & 4,14 & 5 & 4,08 \\
\hline & & & $\mathrm{B}$ & 4,65 & 4,65 & 4,52 & 4,58 & 4,71 & 5 & 4,62 \\
\hline & & \multirow{2}{*}{4} & A & 4,14 & 4,07 & 4,01 & 3,82 & 3,88 & 5 & 3,98 \\
\hline & & & B & 4,65 & 4,58 & 4,52 & 4,33 & 4,45 & 5 & 4,50 \\
\hline & & \multirow{2}{*}{6} & $\mathrm{~A}$ & 3,82 & 3,82 & 3,94 & 3,88 & 3,88 & 5 & 3,86 \\
\hline & & & B & 4,52 & 4,33 & 4,26 & 4,33 & 4,39 & 5 & 4,36 \\
\hline & \multirow{6}{*}{$\stackrel{1}{\mathcal{N}}$} & \multirow{2}{*}{2} & A & 3,82 & 3,82 & 3,88 & 3,94 & 3,75 & 5 & 3,84 \\
\hline & & & $\mathrm{B}$ & 4,52 & 4,52 & 4,26 & 4,20 & 4,33 & 5 & 4,36 \\
\hline & & \multirow{2}{*}{4} & A & 3,69 & 3,82 & 3,82 & 3,75 & 3,69 & 5 & 3,75 \\
\hline & & & B & 4,39 & 4,45 & 4,26 & 4,14 & 4,20 & 5 & 4,28 \\
\hline & & \multirow{2}{*}{6} & $\mathrm{~A}$ & 3,69 & 3,75 & 3,82 & 3,63 & 3,63 & 5 & 3,70 \\
\hline & & & $\mathrm{B}$ & 4,33 & 4,26 & 4,07 & 4,07 & 3,94 & 5 & 4,13 \\
\hline & \multirow{6}{*}{ 은 } & \multirow{2}{*}{2} & A & 3,69 & 3,63 & 3,69 & 3,75 & 3,69 & 5 & 3,69 \\
\hline & & & B & 4,20 & 4,07 & 4,26 & 4,14 & 4,07 & 5 & 4,14 \\
\hline & & \multirow{2}{*}{4} & $\mathrm{~A}$ & 3,63 & 3,56 & 3,69 & 3,43 & 3,63 & 5 & 3,58 \\
\hline & & & B & 4,14 & 4,01 & 3,94 & 4,07 & 3,94 & 5 & 4,02 \\
\hline & & \multirow{2}{*}{6} & A & 3,24 & 3,69 & 3,31 & 3,63 & 3,37 & 5 & 3,44 \\
\hline & & & B & 4,07 & 3,94 & 3,94 & 4,01 & 3,88 & 5 & 3,96 \\
\hline
\end{tabular}

After single and dual component varnish applications of chestnut wood; lowest adhesive resistance was seen in specimens performed single component varnish application and waited for 6 hours in $150{ }^{\circ} \mathrm{C}$, while highest adhesive resistance was seen in specimens performed dual component varnish application and waited for 2 hours in $100{ }^{\circ} \mathrm{C}$ according to heat treatment temperature and time conditions. As a result of comparing adhesive resistance values of non-heat-treated specimens subjected to single component varnish application and heat-treated specimens, specimens waited for 2, 4, 6 hours in $100{ }^{\circ} \mathrm{C}$ have higher adhesive resistance than specimens varnished as without heat treatment, and it was seen that adhesive resistance values decreased in other heat treatment temperatures and times. As a result of comparing dual component varnished non-heat-treated specimens and heat-treated specimens, specimens waited for $2,4,6$ hours in $100{ }^{\circ} \mathrm{C}$ and waited for 2 hours in $125^{\circ} \mathrm{C}$ have higher adhesive resistance, and it was observed that adhesive resistance values decreased in other heat treatment temperatures and times.

\subsection{Brightness Measurements}

Obtained brightness measurement values belong to scotch pine wood were given in Table 8. 
Table 8. Brightness measurement values belong to scotch pine wood

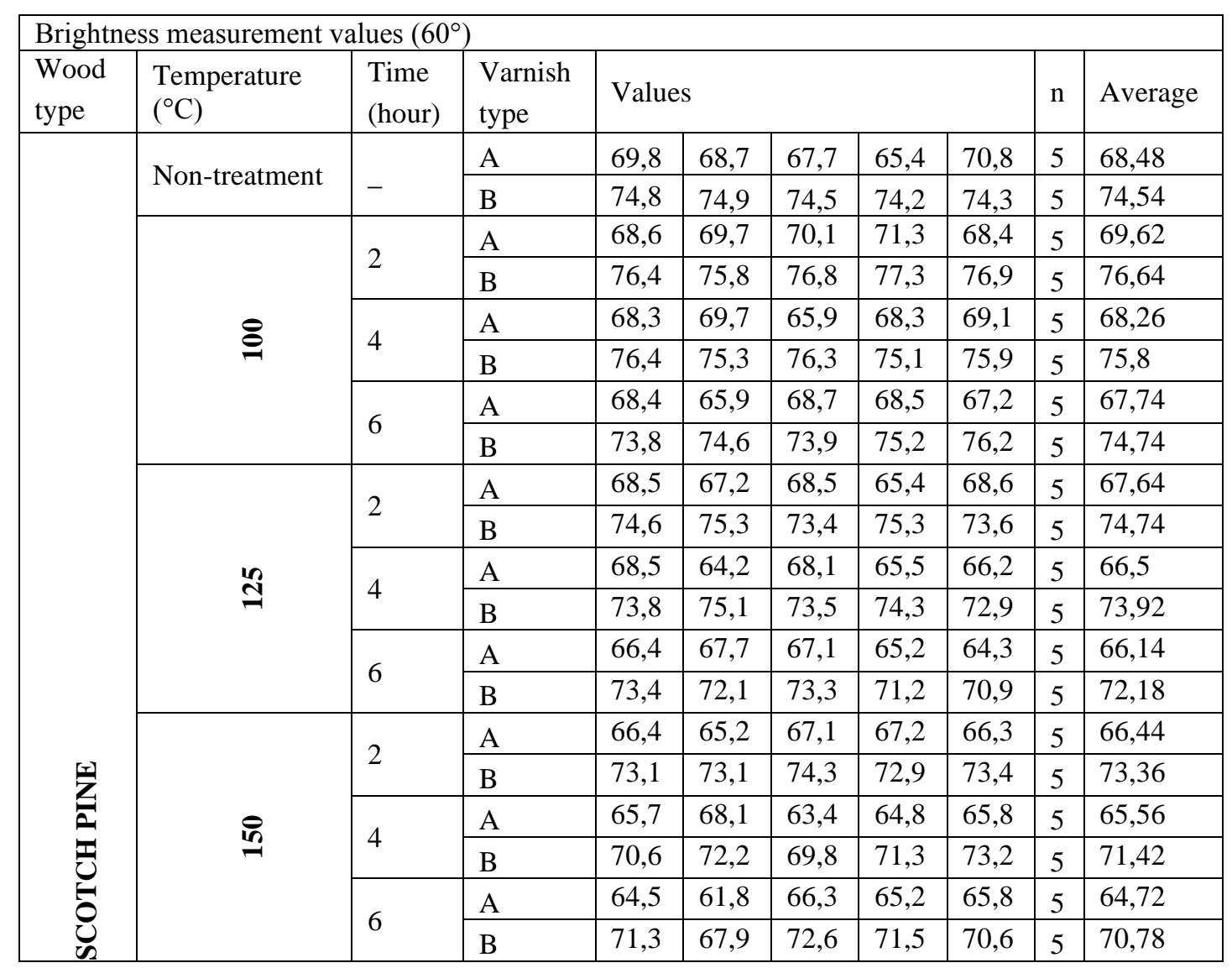

After single and dual component varnish applications of scotch pine wood; lowest brightness values were seen in pieces performed single component varnish application and waited for 6 hours in $150{ }^{\circ} \mathrm{C}$, while highest brightness values were seen in specimens performed dual component varnish application and waited for 2 hours in $100{ }^{\circ} \mathrm{C}$. As a result of comparing brightness values of non-heat-treated specimens subjected to single component varnish application and heat-treated specimens, brightness values of specimens waited for 2 hours in $100{ }^{\circ} \mathrm{C}$ have higher than specimens varnished as without heat treatment, and it was seen that brightness values decreased in other heat treatment temperatures and times. As a result of comparing dual component varnished non-heat-treated specimens and heat-treated specimens, specimens waited for 2, 4, 6 hours in $100{ }^{\circ} \mathrm{C}$ have higher brightness values, and it was observed that brightness values decreased in other heat treatment temperatures and times.Obtained brightness measurement values belong to beech wood were given in Table 9. 
Table 9. Brightness measurement values belong to beech wood

\begin{tabular}{|c|c|c|c|c|c|c|c|c|c|c|}
\hline \multicolumn{11}{|c|}{ Brightness measurement values $\left(60^{\circ}\right)$} \\
\hline $\begin{array}{l}\text { Wood } \\
\text { type }\end{array}$ & \multirow{3}{*}{$\begin{array}{l}\text { Temperature } \\
\left({ }^{\circ} \mathrm{C}\right) \\
\text { Non-treatment }\end{array}$} & \multirow{3}{*}{$\begin{array}{l}\begin{array}{l}\text { Time } \\
\text { (hour) }\end{array} \\
-\end{array}$} & \multirow{2}{*}{$\begin{array}{l}\text { Varnish } \\
\text { type } \\
\text { A }\end{array}$} & \multicolumn{5}{|c|}{ Values } & \multirow{2}{*}{$\begin{array}{l}n \\
5\end{array}$} & \multirow{2}{*}{$\begin{array}{l}\text { Average } \\
66,82\end{array}$} \\
\hline \multirow{20}{*}{ 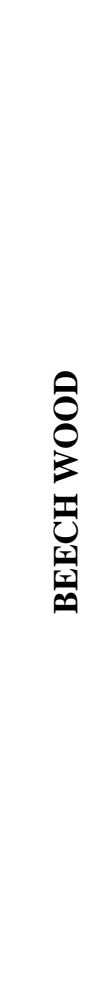 } & & & & 68,3 & 66,4 & 68,1 & 64,9 & 66,4 & & \\
\hline & & & $\mathrm{B}$ & 74,8 & 73,9 & 75,5 & 74,4 & 74,1 & 5 & 74,54 \\
\hline & \multirow{6}{*}{ 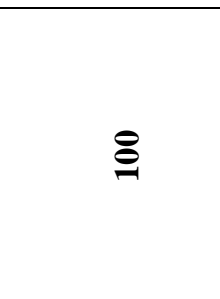 } & \multirow{2}{*}{2} & A & 67,9 & 66,4 & 67,2 & 68,1 & 65,5 & 5 & 67,02 \\
\hline & & & $\mathrm{B}$ & 75,9 & 75,6 & 76,1 & 76,3 & 77,1 & 5 & 76,2 \\
\hline & & \multirow{2}{*}{4} & A & 68,2 & 63,8 & 66,1 & 63,7 & 63,5 & 5 & 65,06 \\
\hline & & & $\mathrm{B}$ & 77,6 & 76,5 & 75,5 & 75,2 & 74,6 & 5 & 75,88 \\
\hline & & \multirow{2}{*}{6} & A & 63,5 & 68,2 & 64,8 & 63,5 & 63,2 & 5 & 64,64 \\
\hline & & & B & 75,2 & 75,6 & 76,1 & 73,4 & 73,8 & 5 & 74,82 \\
\hline & \multirow{6}{*}{$\stackrel{1}{\mathcal{I}}$} & \multirow{2}{*}{2} & A & 63,4 & 66,2 & 65,2 & 64,8 & 63,3 & 5 & 64,58 \\
\hline & & & B & 75,3 & 73,4 & 72,2 & 74,3 & 75,6 & 5 & 74,16 \\
\hline & & \multirow{2}{*}{4} & A & 65,2 & 65,4 & 63,9 & 64,5 & 61,3 & 5 & 64,06 \\
\hline & & & B & 75,5 & 71,3 & 70,6 & 71,2 & 73,1 & 5 & 72,34 \\
\hline & & \multirow{2}{*}{6} & $\mathrm{~A}$ & 63,2 & 60,8 & 61,8 & 62,4 & 61,1 & 5 & 61,86 \\
\hline & & & B & 71,9 & 70,6 & 71,2 & 70,6 & 68,9 & 5 & 70,64 \\
\hline & \multirow{6}{*}{ in } & \multirow{2}{*}{2} & A & 62,4 & 62,8 & 59,1 & 59,7 & 63,8 & 5 & 61,56 \\
\hline & & & B & 71,5 & 70,7 & 69,1 & 70,5 & 71,5 & 5 & 70,66 \\
\hline & & \multirow{2}{*}{4} & A & 61,6 & 61,8 & 60,7 & 59,9 & 59,2 & 5 & 60,64 \\
\hline & & & B & 71,3 & 68,3 & 69,8 & 69,1 & 67,3 & 5 & 69,16 \\
\hline & & \multirow{2}{*}{6} & $\mathrm{~A}$ & 59,7 & 61,2 & 62,8 & 58,3 & 58,4 & 5 & 60,08 \\
\hline & & & B & 70,4 & 67,8 & 68,8 & 65,9 & 67,8 & 5 & 68,14 \\
\hline
\end{tabular}

After single and dual component varnish applications of beech wood; lowest brightness values were seen in pieces performed single component varnish application and waited for 6 hours in $100{ }^{\circ} \mathrm{C}$, while highest brightness values were seen in specimens performed dual component varnish application and waited for 2 hours in $100{ }^{\circ} \mathrm{C}$. As a result of comparing brightness values of non-heat-treated specimens subjected to single component varnish application and heattreated specimens, brightness values of specimens waited for 2 hours in $100{ }^{\circ} \mathrm{C}$ have higher than specimens varnished as without heat treatment, and it was seen that brightness values decreased in other heat treatment temperatures and times. As a result of comparing dual component varnished non-heat-treated specimens and heat-treated specimens, specimens waited for $2,4,6$ hours in $100{ }^{\circ} \mathrm{C}$ have higher brightness values, and it was observed that brightness values decreased in other heat treatment temperatures and times.

Obtained brightness measurement values belong to chestnut wood were given in Table 10. 
Table 10. Brightness measurement values belong to chestnut wood

\begin{tabular}{|c|c|c|c|c|c|c|c|c|c|c|}
\hline \multicolumn{11}{|c|}{ Brightness measurement values $\left(60^{\circ}\right)$} \\
\hline $\begin{array}{l}\text { Wood } \\
\text { type }\end{array}$ & $\begin{array}{l}\text { Temperature } \\
\left({ }^{\circ} \mathrm{C}\right)\end{array}$ & $\begin{array}{l}\text { Time } \\
\text { (hour) }\end{array}$ & $\begin{array}{l}\text { Varnish } \\
\text { type }\end{array}$ & \multicolumn{5}{|c|}{ Values } & $\mathrm{n}$ & Average \\
\hline \multirow{20}{*}{ 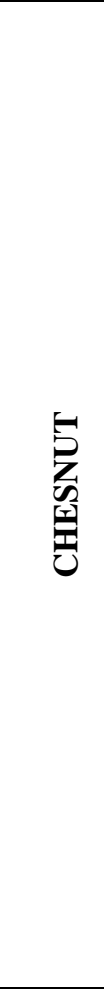 } & \multirow[t]{2}{*}{ Non-treatment } & \multirow[b]{2}{*}{-} & A & 64,4 & 63,3 & 63,6 & 64,3 & 63,9 & 5 & 63,9 \\
\hline & & & $\mathrm{B}$ & 73,4 & 73,1 & 72,3 & 72,3 & 72,5 & 5 & 72,72 \\
\hline & \multirow{6}{*}{ ఠ } & \multirow{2}{*}{2} & A & 65,2 & 64,5 & 62,1 & 66,1 & 64,3 & 5 & 64,44 \\
\hline & & & $\mathrm{B}$ & 72,6 & 75,3 & 74,3 & 75,1 & 74,2 & 5 & 74,3 \\
\hline & & \multirow{2}{*}{4} & A & 68,3 & 64,2 & 63,5 & 64,2 & 63,1 & 5 & 64,66 \\
\hline & & & $\mathrm{B}$ & 73,2 & 72,6 & 71,8 & 74,3 & 72,2 & 5 & 72,82 \\
\hline & & \multirow{2}{*}{6} & $\mathrm{~A}$ & 63,5 & 65,3 & 64,8 & 61,2 & 63,5 & 5 & 63,66 \\
\hline & & & B & 73,5 & 74,3 & 71,5 & 70,6 & 71,3 & 5 & 72,24 \\
\hline & \multirow{6}{*}{$\stackrel{1}{=}$} & \multirow{2}{*}{2} & $\mathrm{~A}$ & 65,2 & 64,3 & 63,8 & 62,5 & 62,8 & 5 & 63,72 \\
\hline & & & B & 71,3 & 72,6 & 68,9 & 70,6 & 72,2 & 5 & 71,12 \\
\hline & & \multirow{2}{*}{4} & A & 65,3 & 62,4 & 60,4 & 61,3 & 61,1 & 5 & 62,1 \\
\hline & & & B & 73,2 & 68,7 & 69,5 & 68,9 & 70,5 & 5 & 70,16 \\
\hline & & \multirow{2}{*}{6} & A & 61,3 & 59,9 & 63,2 & 58,7 & 58,9 & 5 & 60,4 \\
\hline & & & B & 68,3 & 70,8 & 69,7 & 71,2 & 70,1 & 5 & 70,02 \\
\hline & \multirow{6}{*}{ in } & \multirow{2}{*}{2} & A & 60,5 & 61,3 & 61,2 & 61,2 & 60,2 & 5 & 60,88 \\
\hline & & & B & 70,8 & 69,8 & 70,5 & 68,8 & 71,6 & 5 & 70,3 \\
\hline & & \multirow{2}{*}{4} & A & 59,4 & 60,8 & 61,3 & 58,6 & 60,5 & 5 & 60,12 \\
\hline & & & B & 70,4 & 68,9 & 68,8 & 68,9 & 69,4 & 5 & 69,28 \\
\hline & & \multirow{2}{*}{6} & A & 57,9 & 57,7 & 58,4 & 58,2 & 58,1 & 5 & 58,06 \\
\hline & & & B & 68,5 & 67,3 & 67,3 & 68,7 & 68,2 & 5 & 68 \\
\hline & & & & & & & & & & \\
\hline
\end{tabular}

After single and dual component varnish applications of chestnut wood; lowest brightness values were seen in pieces performed single component varnish application and waited for 6 hours in $150{ }^{\circ} \mathrm{C}$, while highest brightness values were seen in specimens performed dual component varnish application and waited for 2 hours in $100{ }^{\circ} \mathrm{C}$. As a result of comparing brightness values of non-heat-treated specimens subjected to single component varnish application and heat-treated specimens, brightness values of specimens waited for 2 and 4 hours in $100{ }^{\circ} \mathrm{C}$ have higher than specimens varnished as without heat treatment, and it was seen that brightness values decreased in other heat treatment temperatures and times. As a result of comparing dual component varnished non-heat-treated specimens and heat-treated specimens, specimens waited for 2 and 4 hours in $100{ }^{\circ} \mathrm{C}$ have higher brightness values, and it was observed that brightness values decreased in other heat treatment temperatures and times.

\section{CONLUSION AND SUGGESTIONS}

Hardness resistance values; increase heat treatment temperature degree and time in all tree species caused an increase of hardness values in single component and dual component varnish type.

Varnishes' surface sticking resistance values; while it was seen improvements in Varnishes' surface sticking resistance in all tree species specimens subjected to heat treatment in $100{ }^{\circ} \mathrm{C}$ for 2,4 , and 6 hours in all tree species, it was seen negativities in adhesive resistances in specimens waited 2,4 , and 6 hours in $125^{\circ} \mathrm{C}$ and $150{ }^{\circ} \mathrm{C}$.

Brightness values; it was seen negativities in brightness values in specimens waited 4 , and 6 hours in $100{ }^{\circ} \mathrm{C}$, and waited 2,4 , and 6 hours in $125^{\circ} \mathrm{C}$ and $150{ }^{\circ} \mathrm{C}$, while it was seen improvements in brightness values in all tree species specimens subjected to heat treatment in $100{ }^{\circ} \mathrm{C}$ for 2 hours in all tree species.

\section{REFERENCES}

ANS/IS01522., (1998)."Paints and Varnishes-Pendulum Damping Test Approved as an American National Standard by ASTM International".

ASTM D 358., (1998). ' Standard Specification for Wood to Be Used as Panels in Weathering Tests of Coatings" American Society for Testing and Materials, 5-9.

ASTM D-4541., (1995). "Standard Test Method for PullOff Strength of Coatings Using Portable Adhesion Testers", American Society for Testing and Materials, 12-15.

Atar, M.,(1999). "Effects on the surface treatment of Coloropening chemical substances in wooden material, Ph. D. Thesis, Gazi University, Graduate School of Natural and Applied Sciences, Ankara,15-17. 
Budakçı, M., (1997). "The eefects of layer thickness in varnish to brillance, hardness and resistance of stick, Master thesis, Gazi University, Graduate School of Natural and Applied Sciences, Ankara.

Highley, T.L., Kicle, T.K.,(1990). Phytopsthology (Blanchette, R.A., et al.).

Sanıvar, N., (2001). Woodworking Surface Finishing, National Education Printing House, İstanbul.

Kantay R., Kartal N., (2007). Heat process practices and properties of heat treated wooden material. Wooden Magazine, 33:36-42.

Ozalp, M., (2008). The investigation of borax pentahydrate influences with double components in varnish applications of wood materials', Wood Res.,53(4): 121-128.

Ozalp, M., Korkut, S., (2011). The research of borax pentahydrate effects with water-based double components in varnish applications of wooden materials', Wood Res., 56(1), : 105-114.

Sogutlu, C., (2004). "The using of some native wood species in kundekari construction. Ph. D. Thesis, Gazi University, Graduate School of Natural and Applied Sciences, 101-102 (1504) Ankara.

Thermodwood Handbook., (2003). Finnish Thermowood Association, Helsinki, Finland.

TS EN 24624., (1996). "Paints and varnishes - Pulling Test", Ankara.

TS 4318 EN ISO 2813., (2002). Paint and varnishesnonmetallic paint films determining the brillance in $20^{\circ}, 60^{\circ}$ and $85^{\circ}, T S E$, Ankara. 